\title{
A SAÚDE NEONATAL FRENTE À SÍNDROME HIPÓXICO ISQUÊMICA: REVISÃO DE LITERATURA
}

\author{
Neonatal Health In The Face Of Hypoxic Ischemic Syndrome: A Literature Review
}

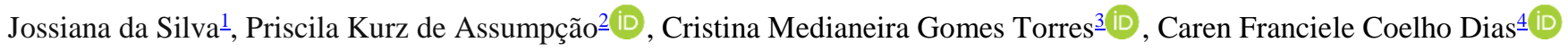

\begin{abstract}
RESUMO
Uma assistência de qualidade é importante para reduzir a mortalidade infantil, dessa forma é necessário estabelecer uma qualidade na assistência tanto das gestantes quanto dos RNs. Este estudo objetivou identificar na literatura cientifica nacional de enfermagem o que vem sendo abordado a respeito da saúde neonatal frente à síndrome hipóxico isquêmica. Trata-se de uma revisão narrativa da literatura. A busca dos dados ocorreu no período de março a abril de 2019, na qual foram realizados por meio da base de dados: Literatura Latino-Americana e do Caribe em Ciências de Saúde e a Base de dados da Enfermagem via Biblioteca Virtual em Saúde utilizando como critérios de inclusão: artigos disponíveis na íntegra e online, gratuitos, no idioma português, com o recorte temporal de 2003 a 2019. Excluiu-se os estudos que não respondem à pergunta da pesquisa. Para seleção dos artigos, foram utilizadas as palavras combinados com operadores booleanos: "Enfermagem" AND "Asfixia Neonatal" AND "Hipóxico-isquêmica Encefálica", resultando inicialmente em 178 estudos, após a aplicação dos critérios de inclusão e exclusão compuseram o corpus da pesquisa seis artigos. A partir da análise dos estudos foi descrita duas categorias: Cuidado da equipe multiprofissional para a prevenção de Síndrome Hipóxia Isquêmica, onde foram abordados o cuidado prestado a mulher e ao recémnascido pela equipe multiprofissional e Enfermagem e a saúde materno-neonatal frente a Síndrome Hipóxia Isquêmica, abordando as vivências de mulheres diante do cuidado de enfermagem materno- neonatal. Após a realização desse estudo, entendeu-se a sua relevância e como é importante haver mais pesquisas voltadas a síndrome hipóxia-isquêmica para uma a qualidade da assistência.

Palavras-chave: Enfermagem, Asfixia neonatal, Recém-nascido, Cuidado perinatal.
\end{abstract}

\section{ABSTRACT}

Quality care is important to reduce infant mortality, so it is necessary to establish quality care for both pregnant women and newborns. This study aimed to identify in the national scientific literature of nursing what has been addressed regarding neonatal health facing the hypoxic-ischemic syndrome. This is a narrative review of the literature. The search for data occurred in the period from March to April 2019, in which they were conducted through the database: Latin American and Caribbean Literature on Health Sciences and the Nursing Database via the Virtual Health Library using as inclusion criteria: articles available in full and online, free of charge, in Portuguese, with the temporal cut from 2003 to 2019. Studies that did not answer the research question were excluded. For selection of articles, the words combined with Boolean operators were used: "Nursing" AND "Neonatal Asphyxia" AND "Hypoxic-ischemic encephalic", resulting initially in 178 studies, after the application of inclusion and exclusion criteria composed the corpus of the research six articles. From the analysis of the studies, two categories were described: Care of the multiprofessional team for the prevention of hypoxic-ischemic syndrome, which addressed the care provided to women and newborns by the multiprofessional team and Nursing and maternal-neonatal health facing hypoxic-ischemic syndrome, addressing the experiences of women facing the maternal-neonatal nursing care. After this study, its relevance was understood and how important it is to have more research focused on hypoxic-ischemic syndrome for a better quality of care.

Keywords: Nursing, Asphyxia neonatorum, Newborn, Perinatal care.

1. Colégio técnico SEG/ Gandhi.

2. Enfermeira, Mestre em Pediatria e Saúde da Criança pela Pontifícia Universidade Católica do Rio Grande do Sul (PUC), RS, Brasil. Faculdade Integrada de Santa Maria (FISMA), Santa Maria, RS, Brasil.

3. Enfermeira pela Faculdade Integrada de Santa Maria (FISMA), Santa Maria, RS, Brasil. Hospital Bruno Born, Lajeado, RS, Brasil.

4. Enfermeira, Mestre em enfermagem pela Universidade Federal de Santa Maria, Santa Maria, RS, Brasil. Hospital Universitário de Santa Maria, Santa Maria (HUSM), RS, Brasil.

*Autor para Correspondência. E-mail: joo-ssiana@hotmail.com 


\section{INTRODUÇÃO}

O período gestacional acarreta em diversas formas de desequilíbrio diante de múltiplas mudanças ocorridas, pois este período é pleno em alterações na vida da mulher, podendo gerar reclamações comuns de desconforto dependendo de cada momento da gestação. Assim, a gravidez é o estágio em que o organismo da mulher passa por diversas modificações fisiológicas e hormonais que ocasionam mudanças, como o crescimento do feto em que ocorre o desenvolvimento demasiado do útero e das mamas, que determina sobrecarga de peso, da coluna e da pelve ${ }^{1}$.

A Organização Mundial de Saúde (OMS) recomenda que os partos cesáreos em relação ao número de partos normais realizados sejam de $15 \%$, pois esses valores apresentam indicação específica de cesariana, isso significa que, existe uma situação real que é fundamental para prevenção da saúde da mãe e do bebê em que o procedimento seja realizado por cirurgia e não por parto natural. $\mathrm{O}$ parto normal é o mais aconselhado pelas políticas de saúde, tendo em vista, ser mais prático e de recuperação mais rápida para a puérpera, sendo a cessaria designada em casos em que o parto normal ofereça riscos comprovados à parturiente e seu bebê $\hat{e}^{2}$.

Desse modo, o período de grande vulnerabilidade da vida é o período neonatal, neste momento aumentam-se os riscos socioeconômicos, ambientais, biológicos e culturais, devendo ser realizado cuidados especiais e qualificados de saúde, incluindo a proteção social e os direitos civis, respeitando o que é estabelecido pelo Estatuto da Criança e do Adolescente e pela Constituição Federal. Assim, realizar ações de promoção, prevenção e assistência à saúde direcionada tanto para a puérpera, quanto para o RecémNascido (RN) é necessário, pois estes sensibilizam as condições de saúde desses indivíduos durante toda a vida ${ }^{3}$.

Neste sentido, há uma preocupação dos casos de RN com Síndrome hipóxico-isquêmico (SHI), pois, esta é uma das causas acentuadas do número de óbitos. A SHI em bebês é responsável por grande número de mortes neonatais nos países em desenvolvimento, os fatores que contribuem para a morbimortalidade perinatal vão desde a deficiência na quantidade e qualidade da assistência pré-natal, atraso da população na procura por assistência médica, manejo inadequado da assistência ao parto e falta de recursos físicos ou intelectuais para fornecer uma adequada reanimação neonatal nas complicações da síndrome hipóxico-isquêmica ${ }^{4}$.

Uma assistência de qualidade é importante para reduzir a mortalidade infantil, o acompanhamento no prénatal de forma adequada contribui para a identificação precoce de riscos e para isso, foi criado o programa Rede
Cegonha que busca a ampliação e qualificação do cuidado à gestante e recém-nascido ${ }^{3}$.

Portanto, é pertinente discutir sobre a qualificação dos profissionais de enfermagem frente à síndrome hipóxicoisquêmica, para a diminuição do número de óbitos e complicações ao recém-nascido. Nesta perspectiva, questiona-se: Como está sendo abordado na literatura cientifica nacional de Enfermagem a Saúde Neonatal frente à Síndrome Hipóxico-Isquêmica? Para responder à questão de pesquisa, este estudo objetivou identificar na literatura cientifica nacional de enfermagem o que vem sendo abordado a respeito da saúde neonatal frente à síndrome hipóxico isquêmica.

\section{REVISÃO DE LITERATURA}

Da concepção da gestação ao parto: considerações e transformações relevantes

O período gestacional para muitas mulheres é o momento mais esperado da vida feminina, quando se inicia a constituição de sua família. Este período repleto de valores e transformações emocionais e físicas vivenciadas por todas, que determinam aspectos relevantes quanto à efetividade do acompanhamento de pré-natal à gestante no qual são ofertadas respostas e apoio emocional no processo da gestação ${ }^{5}$.

Com o intuito de incentivar e aumentar a cobertura de atendimentos a gestantes instituiu-se o Programa de Humanização do Pré-Natal e Nascimento, no qual objetiva desenvolver ações de promoção, prevenção e assistência à saúde das mulheres e recém-nascidos, reduzindo índices de morbimortalidade materna, perinatal e neonatal; aumentando o acesso, a cobertura e a qualidade do cuidado, diminuindo ou evitando deformidades congênitas e malformações fetais a fim de melhorar a assistência ao parto e puerpério ${ }^{3}$.

Para qualificar e objetivar o cuidado integral das mulheres há vários programas e políticas públicas de saúde da mulher durante a gestação, além do Programa de Humanização do Pré-Natal e Nascimento, tem-se a Rede cegonha que consiste em uma tática do Ministério da Saúde para efetuar uma rede de assistência a fim de assegurar o direito ao planejamento reprodutivo e a atenção humanizada ao pré-natal até ao puerpério. Neste contexto a Rede Cegonha realiza o cuidado ao pré-natal; parto e nascimento e puerpério por meio de uma atenção integral e um sistema logístico estruturado em níveis de atenção ${ }^{6}$.

Nesta perspectiva, a enfermagem tem um importante papel no cuidado a gestante e recém-nascido, com base na portaria, trabalha-se com o cuidado humanizado. Tentando 
diminuir as incertezas e anseios das grávidas, uma delas é a escolha do parto e suas dores, visto que a dor na hora do parto não é apenas física e delimitada, mas também aborda o âmbito social, cultural e psíquico da parturiente ${ }^{6}$.

Logo, é necessário que se tenha uma relação de confiança entre à gestante e o profissional de enfermagem para que no final se tenha um parto tranquilo e realizado através de um efetivo pré-natal, sendo que a gestação consiste em um momento de preparação psicológica para a maternidade. Salientando a importância de trabalhar tais temas que devem ser abordados nas consultas de enfermagem no pré-natal; o apoio dos profissionais da saúde durante todo o processo gravídico ajuda na constituição da personalidade materna ${ }^{6}$.

Contudo, no Brasil a referência de cuidado na realização do parto é composta por inúmeras intervenções, contribuindo assim para o crescente número de partos cesáreos e alto índice de morbimortalidade materno-infantil. Os índices de cesariana condizem de $52 \%$ a $88 \%$ na rede privada, para OMS o limite máximo é de $15 \%$, assim uma pesquisa realizada em 24 países nos continentes Africanos, Asiático e América Latina, compondo 372 instituições de assistência ao parto classificaram a taxa de cesariana sem indicação médica entre 0,01 a $2,10 \%{ }^{7}$.

O parto é uma experiência de insegurança e felicidade para a mulher e sua família. Por ser um momento de transformação, no qual não se tem mais o bebê no útero e sim em seus braços, consequentemente, quando experiência do de maneira integral, o parto, propõem novas experiências na vida de todos que estão em torno desse acontecimento. Portanto, é importante preparar gestante para o parto, acompanhando-a durante todo o desenvolvimento do ciclo gravídico, pois assim, evitam-se problemas clínicos e realizase o tratamento adequado quando necessário ${ }^{7}$.

\section{Complicações no parto e o cuidado de enfermagem no puerpério imediato}

A morbimortalidade materna, fetal e neonatal são amplos indicadores de saúde, em 2015, 5,3 milhões de óbitos fetais e neonatais ocorreram e 303 mil óbitos maternos foram registrados no mundo ${ }^{8,9}$. Para tanto, diversos estudos estão relacionando fatores como à morbidade materna com doenças como hipertensão, infecções, hemorragias, além da mortalidade neonatal, baixo peso ao nascer, prematuridade e mortalidade fetal a sobrevida materna e do recém-nascido ${ }^{10}$.

Os sistemas de informações referentes à mortalidade e nascidos vivos não proporcionam a identificação de doenças maternas. Torna-se aliada dos dados de informações hospitalares para que seja possível analisara presença de problemas maternos que podem afetar de maneira negativa os desfechos da gestação e nascimento do bebê $\hat{e}^{10}$.

Com uma consulta pré-natal de qualidade, identificase o início de possíveis fatores de risco maternos, tornando o pré-natal fundamental para um acompanhamento integral desde a unidade básica de saúde até a assistência hospitalar. A identificação precoce dos fatores de risco e das gestações de alto risco demanda conhecimento científico da fisiopatologia obstétrica e a efetivação de um processo de trabalho integral e humanizado, além da capacidade de identificar as condições socioeconômicas e demográficas das parturientes, assim como identificar situações de vulnerabilidade dos neonatos ${ }^{3}$.

Conforme a Portaria $\mathrm{n}^{\mathrm{o}} 1.020$, de 29 de maio de 2013, a definição para uma gestação, parto e puerpério de risco a saúde da mulher indica complicações tanto por doenças pré-existentes ou intercorrências durante a gravidez, parto ou no puerpério, ocasionadas por fatores orgânicos, socioeconômicos e demográficos desfavoráveis. Com isso, é importante que haja um cuidado completo na saúde materna e neonatal ${ }^{3}$.

Assim necessita-se que se tenha um acompanhamento nas consultas de enfermagem, pois elas têm a finalidade de garantir a cobertura e melhoria da qualidade do pré-natal, com a introdução das ações de promoção e prevenção a essas parturientes para que na hora do parto não se tenha nenhuma complicação ${ }^{11}$. É fundamental que o enfermeiro tenha conhecimento e competências para realizar o cuidado a saúde da mãe e bebê.

Um estudo realizado no Canadá com 133 mulheres em período pós-parto imediato identificou-se que nas duas primeiras semanas seguintes ao parto as medidas mais afetadas da qualidade de vida da puérpera foram a capacidade física, o nível de energia e a dor. Já em um hospital que realiza partos humanizados no Ceará, foram identificados como diagnósticos de enfermagem principalmente no puerpério imediato a dificuldade na realização de atividades, privação de sono, dor aguda e o conforto prejudicado ${ }^{12,13}$.

É importante entender que neste período também podem ocorrer complicações que se não forem percebidas em tempo hábil podem acarretar na morbidade e mortalidade materna e neonatal; para o corpo da puérpera voltar as suas condições normais pós-parto carece de ambiência e atendimento especializado ${ }^{14}$. Além das complicações identificadas no período pré-natal, há também as complicações pós-parto como hemorragias, hipertensão arterial e infecção puerperal, ingurgitamento mamário e mastite, podendo também apresentar riscos de 
desenvolvimento de transtornos mentais como depressão pós-parto ${ }^{15}$.

As intercorrências que acontecem com a puérpera durante período do parto podem ser evitadas com uma assistência de enfermagem de qualidade, podendo ajudar a mulher a se recuperar bem e prevenir danos ocasionados no parto. A equipe de enfermagem realiza a assistência direta, individual e integral durante todo período puerperal, realizando um cuidado ao ser humano em todos os ciclos vitais para promoção, prevenção, conforto e qualidade de vida $^{15}$.

\section{Síndrome hipóxico-isquêmica e seus respectivos cuidados de enfermagem}

A SHI equivale-se a suspensão ou diminuição grave das trocas gasosas ao nível da placenta ou dos pulmões, ocasionando em hipoxemia, hipercapnia e hipóxia tecidual com acidose metabólica. Durante o período perinatal, o cérebro pode ser privado de oxigênio por dois importantes mecanismos patogênicos. Hipoxemia, que é diminuição da concentração de oxigênio no sangue e isquemia que é a diminuição da quantidade de sangue que irriga o cérebro e, portanto, em ambos os casos, há menor suprimento de oxigênio para as células, o que limita a produção de energia a níveis abaixo dos requisitos celulares ${ }^{16}$.

Conforme a OMS as três principais causas de morte de RN no mundo são infecções, prematuridade e asfixia perinatal ou complicações no parto que constituem $23 \%$ da mortalidade neonatal global ${ }^{17}$. Além disso, a hipóxia perinatal e suas manifestações neurológicas são a causa mais importante de danos cerebrais e sequelas neurológicas em crianças $^{18,19}$.

Existe muitos motivos para ocorrência da asfixia, esta pode ser causada pela interrupção do fluxo sanguíneo umbilical, por alterações diretas na perfusão placentária; insuficiência da troca de gases pela placenta; perfusão placentária inadequada causada por hipotensão materna, falha na função cardíaca materna; isquemia placentária causada por hipertensão arterial materna; falha do RN ao respirar ao nascer; ou na ocorrência de feto comprometido que não suporta o estresse do trabalho de parto ${ }^{20}$.

Existe uma preocupação em relação à asfixia perinatal, para tanto é importante realizar uma assistência especializada e qualificada para que não aconteçam falhas nas ações de prevenção, no diagnóstico e no tratamento dessa patologia ${ }^{21}$.

A asfixia em bebês é responsável pelas mortes neonatais nos países em desenvolvimento, os fatores que contribuem para a morbimortalidade perinatal vão desde a deficiência na quantidade e qualidade da assistência prénatal, atraso da população na procura por assistência médica, manejo inadequado da assistência ao parto e falta de recursos físicos ou intelectuais para fornecer uma adequada reanimação neonatal nas complicações pós-asfixia. Para isso, é necessário realizar uma reanimação neonatal efetiva e para esse fim, é fundamental que haja grupo de profissionais habilitados ${ }^{21}$.

A hipotermia terapêutica é o tratamento utilizado SHI, apesar disso, não é totalmente efetiva e estima-se que aproximadamente $40-50 \%$ dos recém-nascidos tratados, morrerão ou terão uma incapacidade grave. É reconhecido que o benefício da hipotermia é limitado em RN com encefalopatia muito grave ou no contexto de infecção. Uma das causas pelos quais o resfriamento pode ser efetivo em recém-nascido, é devido à hipotermia ser uma resposta fisiológica, que acontece para impedir uma provável lesão cerebral após hipóxia-isquêmica no nascimento ${ }^{22}$.

A SHI gera alterações nas funções cardíacas e respiratórias, o que faz com que a monitoração após o nascimento deva ocorrer a fim de avaliar a frequência respiratória, frequência cardíaca, saturação arterial de oxigênio e a pressão arterial. A apneia é uma manifestação clínica muito presente em bebês que apresentaram a SIH e que obtiveram como complicação a lesão do Sistema Nervoso Central; os RNs que obtiveram a SHI podem apresentar oligúria, fazendo com que a diurese seja controlada durante vinte quatro horas, a fim de preservar o sistema urinário e planejar o tratamento hídrico para o futuro. Testes como dosagem sérica de sódio, cálcio e potássio, assim como o rastreamento da glicose sanguínea devem ser realizados a fim de averiguar demais complicações no diagnóstico de SHI, o exame radiológico de tórax também deve ser realizado, a fim de verificar o nível de comprometimento e complicações respiratórias ${ }^{4}$.

A realização de um exame clínico minucioso apresenta respostas confiáveis, sendo importante eleger a manutenção das vias aéreas, a circulação e a pressão arterial como prioritárias no atendimento para detectar convulsões e observar os sinais de hipertensão intracraniana, realizando o cuidado mais rápido e eficaz. Entende-se que é importante o suporte disponibilizado pela unidade de terapia intensiva neonatal para os aspectos de correção respiratória, distúrbios metabólicos e hemodinâmicos, e para a realização monitorada do uso de anticonvulsivantes, como também, o efeito neuroprotetor da hipotermia terapêutica, em torno de 3-4 ${ }^{\circ} \mathrm{C}$ abaixo da temperatura corpórea, promovendo o aumento da sobrevivência sem sequelas neurológicas, com menor morbimortalidade ${ }^{23}$. 


\section{MATERIAL E MÉTODOS}

Trata-se de uma revisão narrativa da literatura. $\mathrm{O}$ método utilizado tramitou por uma síntese baseada em uma "leitura flutuante" acerca dos materiais que correspondiam aos critérios de inclusão do estudo, o objetivo primordial da pesquisa é descobrir respostas para problemas mediante a utilização de procedimentos científicos ${ }^{24}$.

A busca dos dados ocorreu no período de março e abril de 2019, o estudo foi realizado na Biblioteca Virtual de Saúde (BVS) na Base de Dados da Literatura LatinoAmericana e do Caribe em Ciências da Saúde (Lilacs) e Banco de dados em Enfermagem (Bdenf). Utilizou-se como critérios de inclusão: artigos disponíveis na íntegra e online, gratuitos, no idioma português, com o recorte temporal de 2003 a 2019, este recorte temporal foi necessário para alcançar um maior número de artigos atuais das bases de dados. Excluiu-se os estudos que não respondem à pergunta da pesquisa.

Para seleção dos artigos, foram utilizadas as palavras combinados com operadores booleanos: "Enfermagem" AND "Asfixia Neonatal" AND "Hipóxico-isquêmica Encefálica".

Na busca realizada, encontrou-se inicialmente 178 estudos disponíveis, mas, ao aplicar o filtro da base de dados e viabilizado 11 estudos disponíveis, foram excluídos cinco artigos por não condizerem com a temática abordada, finalizando assim em seis artigos a serem utilizados para a análise. Logo, nesta pesquisa, foram utilizadas seis publicações, descritos e discutidos nos resultados e discussão.

\section{RESULTADOS}

Para extração das informações dos artigos que compuseram o corpus do estudo elaborou-se uma síntese, conforme demonstrado na Quadro 1, contendo os seguintes itens: artigo, autores, objetivo e resultados.
Quadro 1 - Produções obtidas nas fontes de dados, Rio Grande do Sul, Brasil, 2019

\begin{tabular}{cll}
\hline Artigo & Autores & \multicolumn{1}{c}{ Objetivo } \\
\hline A1 & & \\
& & \\
& & \\
& Santos DSS & $\begin{array}{l}\text { Descrever o perfil } \\
\text { dos resultados das } \\
\text { Ultrassonografias } \\
\text { Transfontanelas. }\end{array}$ \\
& & \\
\hline
\end{tabular}

estudo contemplou 52 prontuários de RN. Quanto ao diagnóstico ultrasson., $63 \%$ dos RNs apresentaram resultado normal. As USGT anormais $(37 \%)$ foram classificadas em cinco tipos de injúrias: Hemorragia Intracraniana (HIC) e Hemorragia Peri intraventricular (HPIV) perfazendo 9\% dos afetados, Encefalopatia Hipoxico-isquêmica (EHI, incluindo Leucomalácia Periventricular) com $17 \%$, Dilatação ventricular (DV) com $9 \%$ e Malformações cerebrais com $2 \%$ da população.

As primigestas representaram $44,16 \%$ das parturientes. As intervenções mais recorrentes foram intervenções mais recorrentes foram amniotomia. A posição horizontal dorsal ocorreu em 12,89\% dos partos. A incidência de episiotomia
foi de $15,52 \%$. Das que não foram foi de $15,52 \%$. Das que não foram submetidas à episiotomia, $36,42 \%$ permaneceram com períneo íntegro, havendo apenas um caso de laceração perineal grave. A asfixia neonatal (Apgar $<7$ ) ocorreu em $0,55 \%$ dos partos.

\begin{tabular}{|c|c|c|}
\hline A3 & $\begin{array}{l}\text { Milbrath VM } \\
\text { Siqueira } \\
\text { HCH } \\
\text { Motta MGC } \\
\text { Amestoy SC }\end{array}$ & $\begin{array}{l}\text { Conhecer o processo } \\
\text { de comunicação } \\
\text { entre equipe de saúde } \\
\text { e família criança com } \\
\text { paralisia cerebral nos } \\
\text { três primeiros anos } \\
\text { de vida. }\end{array}$ \\
\hline A4 & $\begin{array}{l}\text { Milbrath VM } \\
\text { Amestoy SC } \\
\text { Soares DC } \\
\text { Siqueira } \\
\text { HCH }\end{array}$ & $\begin{array}{l}\text { Conhecer as } \\
\text { vivências maternas } \\
\text { sobre a assistência } \\
\text { recebida durante o } \\
\text { trabalho de parto e } \\
\text { parto em que o filho } \\
\text { sofreu asfixia } \\
\text { perinatal grave. }\end{array}$ \\
\hline
\end{tabular}

Os resultados mostraram uma série de ruídos no processo de comunicação entre a equipe de saúde e a família da criança que sofreu asfixia perinatal grave.

Os resultados apontaram aspectos positivos e negativos na assistência recebida durante o processo da parturição. Como positivos emergiram: o diálogo, a confiança e a agilidade no atendimento. Como aspectos negativos destacaram-se: o despreparo do profissional para assistir a mulher no momento da intercorrência durante o trabalho de parto e parto; a desumanização na assistência; e a ausência de um acompanhante durante o processo.

Análise dos dados demonstrou a dificuldade vivenciada por essas dificuldade vivenciada por essas
mulheres/mães com a ruptura entre o parto idealizado e o parto ocorrido, assim como os medos e anseios gerados diante da separação da díade mãe/recém-nascido. Além disso, foi possível constatar falta de harmonia e consonância na conduta dos profissionais de saúde diante dessa complexa situação.

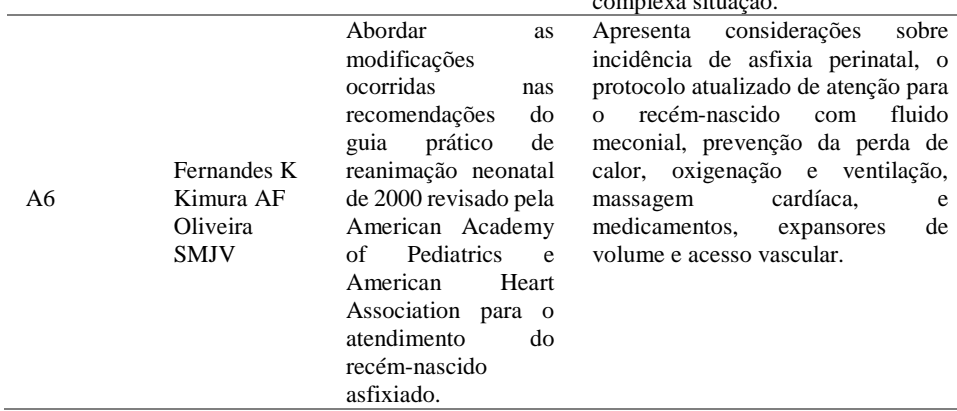

Fonte: Dados coletados pelos autores, 2019. 
Em 2003 foi o primeiro estudo encontrado, após apenas em 2010 foi realizada pesquisa, sendo que este foi o ano com maior incidência de trabalhos realizados com esta temática com um total de duas pesquisas, observou-se também um espaço de tempo não havendo publicação entre 2011 a 2017. Em relação a abordagem metodológica dos estudos, quatro artigos são de abordagem qualitativa. Como instrumento de coleta nas pesquisas qualitativas foram utilizadas as entrevistas semiestruturada e os quantitativos uma busca documental.

Analisou-se que os assuntos abordados da respectiva temática foram: dois artigos tiveram como objetivo conhecer as vivências das mães, um artigo descreve o perfil dos resultados de ultrassonografias transfontanelas, dois artigos analisaram o trabalho da equipe de enfermagem e uma revisão de literatura referente guia prático de reanimação neonatal de 2000. A partir da análise dos estudos foi descrita duas categorias: Cuidado da Equipe Multiprofissional para a Prevenção de Síndrome Hipóxico Isquêmica e Enfermagem e a Saúde Materno-neonatal frente a Síndrome Hipóxia Isquêmica. Assim, busca-se com essa análise um entendimento dos assuntos compreendido nos materiais, baseando a pesquisa, organizando-a de uma forma que a tornará mais compreensível, obtendo uma relação e contraste entre os assuntos com a fundamentação teórica.

\section{DISCUSSÃO}

Cuidado da equipe multiprofissional para a prevenção de Síndrome Hipóxia Isquêmica

Esta categoria foi criada a partir dos estudos A1, A2, A6, onde abordam o cuidado da equipe multiprofissional acerca da prevenção de Síndrome Hipóxia Isquêmica.

A atenção à mulher na gestação e parto consiste em um desafio para a assistência, tanto no que tange à qualidade, como quanto aos princípios subjetivos do cuidado, muitas vezes centrado no modelo tecnocrático, para tanto a atenção multiprofissional tenta minimizar o sofrimento, tornando a vivência do trabalho de parto uma experiência de crescimento e realização para a mulher e sua família. A equipe multiprofissional apresenta características que consiste em flexibilizar a divisão do trabalho, exercer a autonomia profissional e proporcioná-la ao paciente, construir um projeto assistencial comum integrando os agentes envolvidos, no sentido de construírem consensos nos $\operatorname{acordos}^{25}$.

A asfixia perinatal é um agravo ao feto ou ao RN que ocorre com maior frequência nos períodos pré e intraparto, caracterizado por privação de oxigênio (hipóxia) e distúrbio perfusional (isquemia), com repercussões sistêmicas múltiplas. Dentre os órgãos e/ou sistemas afetados pela asfixia destaca-se o sistema nervoso central, cujo envolvimento configura a chamada Encefalopatia HipóxicoIsquêmica ${ }^{26}$.

Com isso, alguns autores afirmam que a equipe multiprofissional necessita adquirir domínio de determinadas técnicas e habilidades comportamentais para atuar com sucesso na assistência. Além de protocolos os exames de imagens como a ultrassonografia contribuem para o cuidado prestado a mães, sendo importante também ao $\mathrm{RN}$ acometido a $\mathrm{SHI}^{21}$.

A ultrassonografia desempenha importante papel no diagnóstico e na identificação de lesões cerebrais em RN de risco, além de prever alterações no neurodesenvolvimento ao longo prazo, com possibilidade de realização à beira do leito utilizando-se aparelhos portáteis, assim se mantém o equilíbrio térmico e hemodinâmico de $\mathrm{RN}$ gravemente enfermos, os profissionais envolvidos no cuidado devem estar atentos aos sinais clínicos. Desse modo, em um estudo utilizando a ultrassonografia foi encontrado que a maioria das injurias cerebrais diagnosticadas são classificadas como Encefalopatia hipóxico isquêmica, incluindo Leucomalácia Periventricular (17\%), seguida de dilatação ventricular e hemorragia intracraniana, ambas envolvendo $9 \%$ da população em estudo ${ }^{27}$.

A asfixia perinatal, a qual pode desenvolver SHI, associada ao parto ainda é uma das principais causas de morbimortalidade neonatal, para diagnosticar utiliza-se índice de Apgar, sendo que o valor do índice de Apgar menor do que 7 no $5^{\text {a }}$ minuto devida passou a ser o referencial mais importante no diagnóstico e no prognóstico. Com isso, a enfermagem junto com a equipe multiprofissional precisa utilizar de técnica desde o nascimento até quando o a recepção dos recém-natos para beneficiar a mãe como a criança ${ }^{28}$.

Identificar as patologias cerebrais mais prevalentes são de grande valia pelo seu poder norteador na prática da assistência ao paciente recém-nascido. Portanto, realizar um trabalho em equipe com atuação interdisciplinar pressupõe trocas significativas, para o diagnóstico e para a realização de cuidados. A diferença de saberes, sob esse enfoque, possibilita ampliar e potencializar os processos interativos e encontrar respostas múltiplas para os problemas de saúde que, na maioria das vezes, também são multifatoriais ${ }^{29}$. 
Enfermagem e a saúde materno-neonatal frente à Sindrome Hipóxia Isquêmica

Esta categoria foi contemplada pelos estudos A3, A4 e A5, abordando como tema principal a enfermagem frente a síndrome hipóxia isquêmica.

Durante o trabalho de parto, parto e período neonatal imediato, são reconhecidos pelo menos cinco mecanismos fundamentais que podem determinar a asfixia em recémnascidos, a falência das trocas gasosas pela placenta; interrupção do fluxo sanguíneo pelo funículo umbilical; perfusão inadequada do lado materno da placenta; feto já comprometido que não tolera a hipóxia transitória de trabalho de parto normal e a impossibilidade do RN inflar seu pulmão e completar com sucesso a transição para a vida extrauterina ${ }^{30}$.

A assistência de enfermagem ao RN necessita ser estruturada e organizada para realizar um melhor atendimento, com recursos especializados. $\mathrm{O}$ papel do enfermeiro diante de um RN com asfixia é realizar um cuidado eficiente, a monitorização dos sinais vitais, sondagens, em um ambiente limpo, via aérea permeável, ventilação adequada, utilizando medidas de reanimação quando necessário, dentre outros cuidados ${ }^{31}$.

Quando há um nascimento de criança com SIH, ocasiona uma desestabilidade familiar. É importante que haja um diálogo entre a equipe de saúde e a família, principalmente para construção de vínculo e apoio emocional nesse momento tão importante para os pais. Nessa perspectiva, autores relatam que a comunicação entre a equipe de saúde e a família de criança com asfixia, nesta pesquisa pode compreender que a comunicação apresentou ruídos que prejudicaram o real entendimento da família com a situação de saúde do recém-nascido ${ }^{32}$.

A comunicação é uma ferramenta importante, onde busca intermediar as relações humanas promovendo a sustentabilidade e a consolidação da autonomia, estimulando o usuário a verbalizar, seus anseios, preocupações e dúvidas, proporcionando um forte vínculo com a equipe de enfermagem ${ }^{33}$.

O cuidado de enfermagem baseado na comunicação com famílias que teve criança com SHI, precisa transmitir as informações de maneira clara conforme a capacidade de compreensão e aceitação dos pais, de uma maneira humanizada sem utilizar termos técnicos. Conforme um estudo, as famílias relataram a falta de entendimento acerca do que estava acontecendo com a criança, junto com apreensão de questionar algo que não entenderam, nas informações fornecidas, levavam as famílias a um desconhecimento do diagnóstico da criança ${ }^{32}$.
A equipe de enfermagem deve estar preparada para oferecer informações, estar aberto e atento às interações com vistas a direcioná-los quanto aos cuidados necessários, assim é essencial que os profissionais tenham conhecimento para orientar e dar apoio à família. O cuidado prestado a essas famílias devem compreender suas crenças, valores, e forma de entender, e a partir disso ir repensando a forma de cuidar esta família na sua integralidade. Tendo a interdisciplinaridade e a integralidade como elo acolhedor, onde a família sente-se segura em relação às informações recebidas ${ }^{34}$.

Diante disso, entender as vivências das mães e familiares em que suas crianças foram acometidas por SHI e asfixia perinatal é importante para buscar melhorar o cuidado prestado, com isso houve duas pesquisas em que abordaram esse assunto. Em um estudo realizado na cidade de Rio Grande na Região Sul (RS), objetivou-se conhecer as vivências das mães sobre o parto em que seu filho sofreu asfixia perinatal grave, as pesquisadoras entenderam que o silêncio dos profissionais, percebido pela parturiente, pode ser mais angustiante e desesperador do que saber que seu filho "sofreu" um problema, mas está sendo acompanhado por profissionais qualificados para atendê-lo na Unidade de Terapia Intensiva Neonatal ${ }^{35}$.

É importante que se tenha a compreensão de que, além do cuidado com a criança, tornava-se importante o cuidado com a família, de modo que suas necessidades também sejam atendidas. Prestar cuidados à mulher, de maneira afetuosa, empática e segura, realizando uma prática assistencial voltada à valorização da mulher, para que essa valorização venha a fortalecê-las no processo de parir e posterior de enfrentamento do nascimento do $\mathrm{RN}$ com SHI. São inúmeras e inesperadas as reações dos pais ao serem informados, que, o filho aguardado e projetado poderá apresentar alterações no seu processo, necessitando de apoio e orientação da equipe de saúde ${ }^{36}$.

A atenção humanizada à parturiente, os profissionais devem permanecer presentes durante todo o trabalho de parto, oferecendo às mulheres o apoio psicológico e emocional na parturição, além de técnicas de relaxamento e massagens, música ou quaisquer outras práticas alternativas que tragam alívio e conforto à gestante. A humanização está voltada para o respeito às individualidades, garantia de tecnologia que promova a segurança do recém-nascido e o acolhimento tanto do recém-nascido quanto da sua família buscando facilitar o vínculo mãe-bebê precocemente ${ }^{37}$.

Em outro artigo, foi encontrado aspectos positivos e negativos na assistência recebida durante o trabalho de parto e parto. Como isso, uma pesquisa descreveu como aspectos 
positivos o diálogo com a parturiente, a confiança no profissional que faria o procedimento, e a agilidade no atendimento. Como aspectos negativos destacaram-se o despreparo do profissional para assistir a mulher no momento da intercorrência durante o trabalho de parto e parto, a desumanização na assistência e a não presença de um acompanhante durante o processo ${ }^{38}$.

A enfermagem tem desempenhado um papel importante no cuidado humanizado às mulheres, favorecendo a fisiologia do parto e introduzindo tecnologias que proporcionam cuidado e conforto à mulher, inserindo em seu atendimento habilidades e competências profissionais, como também a utilização de técnicas e conhecimentos múltiplos e complexos, que possam acompanhar o trabalho de parto, resultando na promoção da saúde da mulher e do bebê $\hat{~}^{39} . \mathrm{Na}$ conduta do tratamento de criança com SHI, é fundamental o acompanhamento, de preferência multiprofissional.

O parto é o momento real em que a mulher se torna mãe, após tanto tempo de espera, imaginando como será este momento, realizar o sonho de ter o filho em seus braços, porém, durante esse período podem ocorrem intercorrências com o RN, situação que exige dos profissionais um cuidado imediato ao bebê, sem esquecer a parturiente em todos os seus aspectos, valorizando-a como um ser humano biopsicossocioespiritual. Nestes casos, a equipe de enfermagem precisa conversar com a mãe sobre o ocorrido, algumas vezes informando aos poucos ${ }^{38,34}$.

No que se refere aos aspectos negativos do parto um estudo aponta que as vivências relatadas foram de despreparo de membros da equipe frente a situação ocorrida gerando insegurança, cuidado desumanizado, falta de um acompanhante $^{38}$. Apesar dos aspectos negativos muitas vezes presenciados pelas parturientes, é importante que a equipe de enfermagem, oriente sobre o andamento do parto e realiza a assistência para minimizar traumas e sofrimento.

A humanização da assistência é a promoção do cuidado integral com vínculo de confiança entre paciente e profissional. A objetividade do olhar biomédico, quando confrontada com a subjetividade do usuário, geralmente propicia uma relação mais centrada na doença do que no doente, na prática de uma medicina curativa e não preventiva. Dessa maneira, a enfermagem vem sendo construída, dentro desta base humanizada de atenção, que busca significado na existência do ser humano, deixando de vê-lo apenas como uma doença, e ouvindo mais, procurando atender todas as demandas que cada um traz ao profissional ${ }^{40}$.

Tendo em vista a humanização no parto, há vários benefícios na presença do acompanhante durante o parto, como: diminuição da duração do trabalho de parto, menor necessidade de medicação ou analgesia, e parto operatório ou instrumental, sentimento de confiança, além de uma menor vulnerabilidade à violência obstétrica. $\mathrm{O}$ apoio contínuo de um acompanhante no trabalho de parto e nascimento é uma intervenção segura e altamente eficaz para melhorar os resultados maternos e neonatais, com altos índices de satisfação materna, baixo custo, sendo também um direito da mulher ${ }^{41}$. Portanto, existe uma necessidade de capacitação da equipe não somente de enfermagem, mas equipe multiprofissional, para realização do cuidado materno-neonatal diante a síndrome hipóxia isquêmica.

\section{CONSIDERAÇÕES FINAIS}

Os resultados evidenciaram a necessidades de mais pesquisas voltadas a síndrome hipóxico-isquêmica para mapear as carências dessa temática. $\mathrm{O}$ número reduzido de artigos relacionados a esse assunto foi considerado um fator limitante desse estudo, além de não ser uma pesquisa de campo, o que poderia ser avaliado in loco a ação da equipe de enfermagem frete a SHI. Porém, é importante reforçar a ampliação dessa pesquisa para que se tenha a efetiva realização do trabalho.

O profissional enfermeiro mostra-se dotado de habilidades e competências para realizar o cuidado, favorecendo a segurança do paciente RN. Considerando a importância do profissional enfermeiro na motivação e compromisso em promover as mudanças, necessárias relacionadas a comunicação, no cuidado humanizado e de qualidade.

Os resultados surpreenderam em relação ao espaço temporal entre uma publicação e outra, porém não surpreendeu a questão da dificuldade de encontrar pesquisas voltadas ao tema, principalmente em relação ao cuidado prestado em RN com SHI. No entanto, após a conclusão desse estudo e muitas leituras sobre o tema, é possível afirmar que quanto mais for difundida os conceitos de cuidado, menor será a incidência desse fato.

Nesse sentido, percebe-se a importância em investir em processos educativos e em estratégias que são utilizadas nos diversos tipos de práticas. Esta pesquisa é apenas o começo dessa discussão, o que, acredita-se, além de auxiliar na prática da enfermagem, poderá auxiliar no trabalho do enfermeiro, pois instiga a reflexão sobre como está sendo realizado o seu próprio trabalho.

\section{REFERÊNCIAS}

1. Costa E.S., Pinon G.M.B., Costa T.S., Santos R.C.A., et al. Alterações fisiológicas na percepção de mulheres durante a gestação. Rev. Rene, 2010 , 11(2): 86-93. Disponível em: <

http://periodicos.ufc.br/rene/article/view/4531/3414> Acesso em: 28 abr. 2019.

2. Patah L.E.M., Malik A.M. Models of childbirth care and cesarean rates in different countries Rev. Saúde Publica, 2011, 45(1): 185-94. Disponível em: <https://www.scielo.br/j/rsp/a/rMnhFmBRjDPQhkSV3HBgQYH/?lang=en\&fo rmat=pdf $>$ Acesso em: 19 abr. 2019

3. Brasil. Ministério da Saúde. Secretaria de Atenção à Saúde. Departamento de Atenção Básica. Atenção ao pré-natal de baixo risco/Ministério da Saúde. Secretaria de Atenção à Saúde. Departamento de Atenção Básica. Brasília: Editora do Ministério da Saúde, 2012

4. Procianoy R.S., Silveira R.C. Síndrome hipóxico-isquêmica. Jornal de Pediatria, 2001, 77(1): 63-70, 2001. 
5. Souza V.B., Roecker S., Marcon S.S. Ações educativas durante a assistência pré-natal: percepção de gestantes atendidas na rede básica de Maringá-PR.

Rev. Eletr. Enf., 2011, 13(2): 199-210.

doi: https://doi.org/10.5216/ree.v13i2.10162

6. Brasil. Ministério da Saúde. Departamento de Atenção Básica. Rede Cegonha. 2018. Disponível em:

<http://dab.saude.gov.br/portaldab/ape_redecegonha.php>. Acesso em: 28 ago. 2019 .

7. Gomes A.R.M., Pontes D.S., Pereira C.C.A., Brasil A.O.M., et al. Assistência de enfermagem obstétrica na humanização do parto normal. Revista Recien, 2014, 4(11): 23-7. doi: https://doi.org/10.24276/rrecien2358$\underline{3088.2014 .4 .11 .23-27}$

8. World Health Organization; United Nations Children's Fund; United Nations Population Fund; World Bank Group; United Nations. Trends in maternal mortality: 1990 to 2015. Estimatesby WHO, UNICEF, UNFPA, World Bank Groupandthe United Nations Population Division. Geneva: World Health Organization; 2015.

9. Lawn J.E., Blencowe H., Waiswa P., Amouzou A., et al. Stillbirths: rates, riskfactors, and acceleration to wards 2030. Lancet, 2016, 6(387): 587-603. doi: 10.1016/S0140-6736(15)00837-5

10. Moura B.L.A., Alencar G.P., Silva Z.P., Almeida M.F. Internações por complicações obstétricas na gestação e desfechos maternos e perinatais, em uma coorte de gestantes no Sistema Único de Saúde no Município de São Paulo, Brasil. Cad. Saúde Pública, 2018, 34(1). doi: 10.1590/0102 $311 \times 00188016$

11. Brasil. Ministério da Saúde. Secretaria de Atenção à Saúde. Departamento de Ações Programáticas Estratégicas. Atenção à saúde do recém-nascido: guia para os profissionais de saúde/Ministério da Saúde, Secretaria de Atenção à Saúde, Departamento de Ações Programáticas Estratégicas. 2. ed. Brasília: Ministério da Saúde, 2012.

12. Baghirzada, Leyla, Downey, Kristy N., Macarthur, Alison J. Assessment of quality of life indicators in the post partum period. International Journal of Obstetric Anesthesia, 2013, 22(3): 209-16. doi

https://doi.org/10.1016/j.ijoa.2013.03.010

13. Barbosa E.M.G., Oliveira F.D.M., Guedes M.V.C., Monteiro A.R.M., et al. Nursing care for one puerpera base do the theory of comfort. Rev Min Enferm, 2014, 18(4). doi: https://doi.org/10.5935/1415-2762.20140062

14. Garcia E.S.G.F., Leite E.P.R.C., Nogueira D.A. Nursing care to puerpera women in primary care units. Revista de Enfermagem da UFPE, 2013, 7(10): 5923-28 doi: 10.5205/reuol.4377-36619-1-ED.0710201312

15. Cabral R.W.L., Medeiros A.L., Santos S.R. Assistência de enfermagem à mulher no período puerperal: proposta de sistematização. In: VII Congresso brasileiro de enfermagem obstétrica e neonatal, 2011, Belo Horizonte.

Fortalecendo redes e alianças estratégicas pela cidadania e saúde das mulheres e dos recém-nascidos. Belo Horizonte: ABENFO-MG, 2011.

16. James A., Cherian S. Pathophysiology of perinatal hypoxia-ischaemia. Paediatrics and Child Health, 2010, 20(8):351-5. doi: 10.1016/ i.paed.2010.03.002

17. Kurinczuk J., White-Koning M., Badawi N. Epidemiology of neonatal encephalopathy and hypoxic-ischaemic encephalopathy, 2010, 86(6):329-38. doi: 10.1016/j.earlhumdev.2010.05.010

18. Azzopardi D. Clinical management of the baby with hypoxic ischaemic encephalopathy, 2010, 86(6): 345-50. doi:

https://doi.org/10.1016/j.earlhumdev.2010.05.008

19. Delfino A., Weinberger M., Delucchi G., Campo S., et al. Seguimiento de recién nacidos con asfixia perinatal. Arch. Pediatr. Urug., 2010, 81(2): 73-7. Disponível em: 〈http://www.scielo.edu.uy/pdf/adp/v81n2/v81n2a02.pdf〉 Acesso em: 14 abr. 2019.
20. Febrasco. Federação brasileira das associações de ginecologia e obstetrícia. Manual de perinatologia. São Paulo: Federação brasileira das associações de ginecologia e obstetrícia, 2013.

21. Fernandes K., Kimura A.F., Oliveira S.M.J.V. Assistência imediata ao recém-nascido asfixiado: revisão das recomendações do guia prático de reanimação neonatal. Rev. Min. Enf., 7(2):152-5, 2003. Disponível em <https://cdn.publisher.gn1.link/reme.org.br/pdf/v7n2a12.pdf >. Acesso em: 14 abr. 2019.

22. Jacobs S.E., Berg M., Hunt R., Tarnow-Mordi W.O., et al. Cooling for newborns with hypoxic ischaemic encephalopathy.Cochrane Database Syst Rev., 2013, (1): CD003311. doi: 10.1002 / 14651858.CD003311.pub3

23. Rocha M.E.M.O., Resende A.K.M., Araújo R.M., Ferreira R.S.A Assistência de enfermagem na encefalopatia hipóxico-isquêmica (EHI) em uma maternidade de referência estadual. In: Anais do Congresso Brasileiro de Enfermagem Obstétrica e Neonatal. Anais. Campo Grande (MS) CCARGC, 2018. Disponível em: <https//www.even3.com.br/anais/cobeon/63336ASSISTENCIA-DE-ENFERMAGEM-NA-ENCEFALOPATIA-HIPOXICOISQUEMICA-(EHI)-EM-UMA-MATERNIDADE-DE-REFERENCIAESTADUAL>Acesso em: 30 ago. 2018

24. Gil A.C. Como Classificar as Pesquisas? Como Elaborar Projetos de Pesquisa. $5^{\circ}$ Ed. São Paulo: Atlas, 2010.

25. Duarte M.L.C., Boeck J.N. O trabalho em equipe na enfermagem e os limites e possibilidades da estratégia saúde da família. Trab. Educ. Saúde, 2015, 13(3): 709-20. doi: http://dx.doi.org/10.1590/1981-7746-sip00054

26. Fonseca L.F., Xavier C., Pianetti G. Compêndio de Neurologia Infantil. 2a ed. Rio de Janeiro: Medbook; 2011.

27. Santos D.S.S., Prado M.S.G. Occurrence of neurological injuries in newborns diagnosed by transfontanellar ultrasound. Rev. enferm. UFPE online, 2017, 11(Supl. 10): 4081-8. doi: 10.5205/reuol.10712-95194-3 SM.1110sup201710

28. Reis C.S.R., Souza D.O.M., Nogueira M.F.H., Progianti J.M., et al. Analysis of births attended by nurse midwives under the perspective of humanization of childbirth. Rev. Fund. Care Online. 2016, 8(4): 4972-9. doi: 10.9789/2175-5361.2016.v8i4.4972-4979

29. Backes D.S., Carpes A. D., Piovesan C., Haeffner L.S.B., et al. Trabalho em equipe multiprofissional na saúde: da concepção ao desafio do fazer na prática. Disciplinarum Scientia, 2014, 15(2): 277-89.

30. Segre C.A.M., Costa H.P.F., Lippi U.G. Asfixia perinatal "in" perinatologia fundamentos e prática. $3^{\circ}$ ed., São Paulo: Sarvier, 2015

31. Universidade Federal de Santa Catarina. Eventos Agudos Na Atenção Básica- Asfixia. Ed. UFSC- 2013. Disponível em <www.unasus.ufsc.br〉 Acesso em 05 mai. 2019.

32. Milbrath V.M., Siqueira H.C.H., Motta M.G.C., Amestoy S.C. Comunicação entre a equipe de saúde e a família da criança com asfixia perinatal grave. Texto contexto enferm., 2011, 20(4): 726-34.

33. Andrade G.B., Pedroso V.S.M., Weykamp J.M., Soares L.S., et al. Palliative Care and the Importance of Communication Between Nurse and Patient, Family and Caregiver. Rev Fund Care Online, 2019, 11(3):713-17. doi: http://dx.doi.org/10.9789/2175-5361.2019.v11i3.713-717

34. Caneco E.O.V., Milbrath V.M., Freitag V.L., Amestoy S.C. Cuidado prestado pela equipe de saúde às famílias de crianças que sofreram asfixia perinatal grave. Santa Maria, 2017, 43(3): 1-10.

35. Milbrath V.M.; Amestoy S.C., Soares D.C., Siqueira H.C.H. Asfixia perinatal grave: a vivencia materna no momento do parto. Cienc. Cuid. Saúde, 2010, 9(1), p-123-8. doi: https://doi.org/10.4025/cienccuidsaude.v9i1.10538

36. Dantas, M.S.A., Pontes J.F., Assis W.D., Collet N. Facilidades e dificuldades da família no cuidado à criança com paralisia cerebral. Rev Gaúcha Enferm, 2012, 33(3): 73-80. Disponível em: 
<https://www.scielo.br/j/rgenf/a/bSWy5FjftgPHZy99pNGqJvw/?format=pdf\&1 ang=pt>. Acesso em: 2 abr. 2019.

37. Pinheiro B.C., Bittar C.M.L. Expectativas, percepções e experiências sobre o parto normal: relato de um grupo de mulheres. Fractal, Rev. Psico, 2013, 25(3): 585-602. doi: 10.1590/S1984-02922013000300011

38. Milbrath V.M., Amestoy S.C., Soares D.C., Siqueira H.C.H. Vivências maternas sobre a assistência recebida no processo de parturição. Esc Anna Nery (impr.), 2010, 14(3): 462-7.

39. Silva I.A., Silva P.S.F., Andrade E.W.O.F., Moraes F.F., et al. Percepção das puérperas acerca da assistência de enfermagem no parto humanizado. Revista Uningá, 2017, 53(2): 37-43. Disponível em:

<http://revista.uninga.br/index.php/uninga/article/view/1440>. Acesso em: 29 jul. 2019.

40. Morais K.C.P., Botton A., Fruet I.M.A. "Cuidar é simplesmente olhar pra um mundo que você não vê": relatos sobre a humanização em uma residência multiprofissional. Rev. Psicologia em Foco, 2015, 7(10): 54-68.

41. D’Orsi E., Brüggemann O.M., Diniz C.S.G., Aguiar J.M., et al. Social inequalities and women's satisfaction with childbirth care in Brazil: a national hospital-based survey. Cad. Saúde Pública, 2014, 30(Supl. 1): 154-68.

doi: https://doi.org/10.1590/0102-311X00087813 